\title{
Sperm divergence in a passerine contact zone: indication of reinforcement at
}

\section{the gametic level}

Running title: Sperm divergence in a passerine contact zone

Authors: Tomáš Albrechtt ${ }^{1,2}$, Kamila Opletalová ${ }^{1}$, Jiří Reif ${ }^{3}$, Václav Janoušek ${ }^{1}$, Lubomír Piálek $^{4}$, Emily R.A. Cramer ${ }^{5}$, Arild Johnsen ${ }^{5}$, Radka Reifová ${ }^{1}$

${ }^{1}$ Charles University in Prague, Faculty of Science, Department of Zoology, Viničná 7, Praha 2

CZ-12844, Czech Republic; ${ }^{2}$ Institute of Vertebrate Biology, v.v.i., The Czech Academy of Sciences, Květná 8, Brno CZ-60365, Czech Republic; ${ }^{3}$ Charles University in Prague, Faculty of Science, Institute for Environmental Studies, Benátská 2, Praha 2 CZ-12100, Czech Republic; ${ }^{4}$ University of South Bohemia, Faculty of Science, Department of Zoology, Branišovská 1760, České Budějovice CZ-370 05, Czech Republic; ${ }^{5}$ Natural History Museum, University of Oslo, 0318 Oslo, Norway

Correspondence: Tomáš Albrecht albrecht@ivb.cz

Authorship contributions: TA, RR and AJ conceived and designed the study; JR, TA, KO, ERAC, RR collected the data; KO analysed sperm data; TA carried out statistical analyses; LP, VJ and RR performed genetic analyses; TA and RR drafted the manuscript. All the authors contributed to the revision of the manuscript and gave final approval for its publication. 
Acknowledgements: We would like to thank Pavel Kverek, Camille Sottas, Abel Souriau, Hana Kohoutová, Martin Jiran and Paweł Dolata for help with data collection and EMBL Genomics Core Facility for technical support. This study was funded through Czech Science Foundation grants no. $15-10884 \mathrm{Y}, 18-14325 \mathrm{~S}$ to $\mathrm{RR}$ and $\mathrm{TA}$, the Charles University grant PRIMUS/19/SCI/08 to RR, and Norwegian Research Council grant no. 213592 to AJ. Computational resources were provided by the CESNET LM2015042 and the CERIT Scientific Cloud LM2015085, provided under the programme "Projects of Large Research, Development, and Innovations Infrastructures", and ELIXIR CZ research infrastructure project (MEYS Grant No: LM2015047).

Data accessibility: Upon acceptance, all data will be made available on Dryad.

Conflict of Interest: We declare no conflict of interest 